\title{
Quasi-variational inequalities, generalized Nash equilibria, and multi-leader-follower games
}

\author{
Jong-Shi Pang • Masao Fukushima
}

Published online: 11 March 2009

(C) Springer-Verlag 2009

\section{Erratum to: Comput Manage Sci (2005) 2:21-56 DOI 10.1007/s10287-004-0010-0}

\begin{abstract}
In Pang and Fukushima (Comput Manage Sci 2:21-56, 2005), a sequential penalty approach was presented for a quasi-variational inequality (QVI) with particular application to the generalized Nash game. To test the computational performance of the penalty method, numerical results were reported with an example from a multileader-follower game in an electric power market. However, due to an inverted sign in the penalty term in the example and some missing terms in the derivatives of the firms' Lagrangian functions, the reported numerical results in Pang and Fukushima (Comput Manage Sci 2:21-56, 2005) are incorrect. Since the numerical examples of this kind are scarce in the literature and this particular example may be useful in the future research, we report the corrected results.
\end{abstract}

In Subsection 5.3 of Pang and Fukushima (2005), to test the computational performance of the proposed penalty method for a quasi-variational inequality (QVI), numerical experiments were carried out on an example from a multi-leader-follower game in an electric power market. However, due to an inverted sign in the penalty term

The online version of the original article can be found under doi:10.1007/s10287-004-0010-0.

\section{J.-S. Pang}

Department of Industrial and Enterprise Systems Engineering, University of Illinois, 117 Transportation Building, 104 South Mathews Avenue, Urbana, IL 61801, USA e-mail: jspang@uiuc.edu

M. Fukushima $(\varangle)$

Department of Applied Mathematics and Physics, Graduate School of Informatics, Kyoto University, Kyoto 606-8501, Japan e-mail: fuku@i.kyoto-u.ac.jp 
Table 1 Firms' sales and nodal prices. (cf. Tables 2 and 3 in

Pang and Fukushima 2005)

\begin{tabular}{lllll}
\hline Node $i$ & Node $j$ & Firm & Sales $s_{i j}^{f}$ & Price $p_{i}$ \\
\hline 1 & & & & 28.74 \\
2 & & & & 28.07 \\
3 & 1 & 1 & 43.54 & 27.74 \\
1 & 2 & 1 & 28.14 & \\
1 & 3 & 1 & 28.33 & \\
1 & 1 & 1 & 26.87 & \\
2 & 2 & 1 & 11.47 & \\
2 & 3 & 1 & 11.66 & \\
2 & 1 & 2 & 43.54 & \\
2 & 2 & 2 & 28.14 & \\
2 & 3 & 2 & 28.33 & \\
2 & 1 & 2 & 26.87 & \\
3 & 2 & 2 & 11.47 & \\
3 & 3 & 2 & 11.66 & \\
3 & & & & \\
\hline
\end{tabular}

in the example and some missing terms in the derivatives of the firms' Lagrangian functions, the reported numerical results in Pang and Fukushima (2005) are incorrect.

First, on p. 52, firm $f$ 's penalized problem should be written as

$$
\begin{array}{ll}
\operatorname{maximize} & \theta_{f}\left(s^{-f}, s^{f}, w\right)-\frac{1}{2 \rho_{k}} \sum_{i, j \in \mathcal{N}} \max \left(0, u_{i j}^{k, f}+\rho_{k}\left(p_{j}\left(S_{j}\right)-p_{i}\left(S_{i}\right)-w_{i j}\right)\right)^{2} \\
\text { subject to } & \sum_{j \in \mathcal{N}} s_{i j}^{f} \leq \mathrm{CAP}_{i}^{f}, \quad \forall i \in \mathcal{N} \\
& s_{i j}^{f} \geq 0, \quad \forall(i, j) \in \mathcal{N} \times \mathcal{N},
\end{array}
$$

where

$$
\theta_{f}\left(s^{-f}, s^{f}, w\right)=\sum_{j \in \mathcal{N}}\left[p_{j}\left(S_{j}\right) \sum_{i \in \mathcal{N}} s_{i j}^{f}\right]-\sum_{i, j \in \mathcal{N}} w_{i j} s_{i j}^{f}-\sum_{i \in \mathcal{N}}\left(c_{i}^{f} \sum_{j \in \mathcal{N}} s_{i j}^{f}\right) .
$$

Next, the LCP shown on the same page should be written as follows: For all $f \in \mathcal{F}$ and $(i, j) \in \mathcal{N} \times \mathcal{N}$,

$$
\begin{aligned}
& 0 \leq s_{i j}^{f} \perp c_{i}^{f}+e_{i j}-v_{i j}-p_{j}\left(S_{j}\right)+\frac{P_{j}^{0}}{Q_{j}^{0}} \sum_{l \in \mathcal{N}} s_{l j}^{f}+\mu_{i}^{f}-\frac{P_{j}^{0}}{Q_{j}^{0}}\left(\sum_{l \in \mathcal{N}} \lambda_{l j}^{k, f}-\sum_{m \in \mathcal{N}} \lambda_{j m}^{k, f}\right) \geq 0 \\
& 0 \leq \mu_{i}^{f} \perp \mathrm{CAP}_{i}^{f}-\sum_{l \in \mathcal{N}} s_{i l}^{f} \geq 0
\end{aligned}
$$




$$
\begin{aligned}
& 0 \leq v_{i j} \perp \sum_{t \in \mathcal{F}} s_{i j}^{t} \geq 0 \\
& 0 \leq \lambda_{i j}^{k, f} \perp \lambda_{i j}^{k, f}-u_{i j}^{k, f}-\rho_{k}\left(p_{j}\left(S_{j}\right)-p_{i}\left(S_{i}\right)-e_{i j}+v_{i j}\right) \geq 0
\end{aligned}
$$

This example was solved with the same data as those shown in Table 1 of Pang and Fukushima (2005) and the same settings as those described on p. 53 of Pang and Fukushima (2005). After 7 iterations, the termination criterion was satisfied. The obtained results are shown in the table. The ISO's shipping charges $w_{i j}$ were all equal to $e_{i j}$. Moreover, the same problem was solved with the alternative penalty update rule $\rho_{k+1}:=2 \rho_{k}$. Then the same solution was obtained after 19 iterations.

Finally, as in Pang and Fukushima (2005), firm II's costs $c_{i}^{\mathrm{II}}$ were changed to 20 at all 3 nodes. Then the problem was successfully solved with either of the penalty update rules $\rho_{k+1}:=10 \rho_{k}$ and $\rho_{k+1}:=2 \rho_{k}$. The two update rules produced the same solution satisfying the optimality conditions for the original problem.

Acknowledgments The authors thank Koichi Nabetani of Kyoto University, who pointed out inaccuracies in Pang and Fukushima (2005) and provided the numerical results presented here.

\section{Reference}

Pang JS, Fukushima M (2005) Quasi-variational inequalities, generalized Nash equilibria, and multileader-follower games. Comput Manage Sci 2:21-56 